OAI-PMH: http://www.indteca.com/ojs/index.php/Revista Scientific/oai

Ensayo Original / Original Essay

\title{
El pensamiento computacional en la vida cotidiana
}

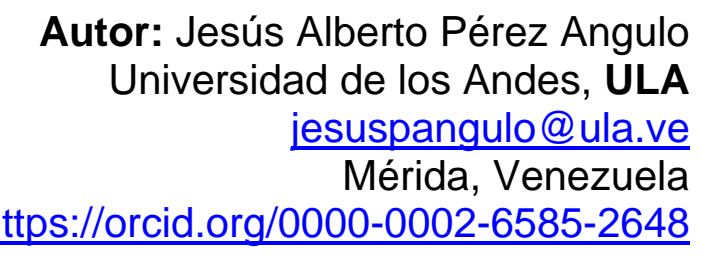

\section{Resumen}

El pensamiento computacional es un concepto relativamente nuevo que surge a principios de este siglo y rápidamente se ha popularizado debido a su premisa principal: todas las personas pueden utilizar habilidades propias del ámbito de la computación para la resolución de problemas en otros ámbitos. Este concepto es relevante porque la resolución de problemas es una de las habilidades necesarias del siglo XXI. Actualmente, se mantiene la idea de que el desarrollo del pensamiento computacional está directamente relacionado con el aprendizaje de la programación de computadores, por tal motivo, los esfuerzos para promover el pensamiento computacional se han orientado hacia la inclusión de cursos introductorios de programación en los currículos de los sistemas educativos. No obstante, considerando que el aprendizaje de la programación es ampliamente conocido por presentar diversas dificultades, es necesario reflexionar sobre otras maneras para el desarrollo del pensamiento computacional. En ese sentido, el propósito de este ensayo es presentar reflexiones que conducen a una propuesta basada en la metacognición, para el desarrollo del pensamiento computacional durante la ejecución de actividades de la vida cotidiana.

\section{cotidiana.}

Palabras clave: pensamiento; habilidad; resolución de problemas; vida

Fecha de Recepción: 08-01-2019
Fecha de Aceptación: 22-04-2019
Fecha de Publicación: 05-08-2019 


\title{
Computational thinking in everyday life
}

\begin{abstract}
Computational thinking is a relatively new concept that emerged at the beginning of this century and has quickly become popular motivated to its main premise: all people can use computer skills to solve problems in other areas. This concept is relevant because solving problems is one of the necessary skills of the 21 st century. Currently, the idea that the development of computational thinking is directly related to the learning of computer programming is maintained, for this reason, the efforts to promote computational thinking have been oriented towards the inclusion of introductory programming courses in the curricula of educational systems. However, considering that learning programming is widely known for presenting various difficulties, it is necessary to reflect on other ways for the development of computational thinking. In this sense, the purpose of this essay is to present reflections that lead to a proposal based on metacognition, for the development of computational thinking during the execution of activities of daily life.
\end{abstract}

Keywords: thinking; skills; problem solving; everyday life.

Date Received: 08-01-2019
Date Acceptance: 22-04-2019
Date Publication:

05-08-2019 


\section{Introducción}

El pensamiento computacional es un concepto que fue planteado por primera vez en el año 2006, y a partir de ese momento, ha venido ganando popularidad debido a su prometedor impacto para todas las personas, ya que su premisa principal es que conceptos asociados al ámbito de la computación pueden ser utilizados para la resolución de problemas en otros ámbitos, y según Trilling y Fadel (2012): "Muchos consideran que la resolución de problemas es la nueva base del aprendizaje del siglo XXI" (pág. 50). Por esa razón, es relevante reflexionar sobre el pensamiento computacional.

Como el pensamiento computacional se basa en resolver problemas haciendo uso de conceptos fundamentales de la computación, según Compañ, Satorre, Llorens y Molina (2015) "Nada mejor para desarrollar la habilidad de resolver problemas usando conceptos informáticos que una asignatura de introducción a la programación" (pág. 1). En ese orden de ideas, según Ortega y Brouard (2018): "La programación está incluyéndose en los currículos educativos de todo el mundo para desarrollar el pensamiento computacional" (pág. 129).

El inconveniente de promover el desarrollo del pensamiento computacional con los cursos introductorios de programación está relacionado a las dificultades que son propias del aprendizaje de la programación. Por ejemplo, en el ámbito universitario, Pérez y Castro (2018a), indican que el razonamiento lógico: "ha sido detectado como una debilidad de la mayoría de los estudiantes" (pág. 8); Pérez y Pedroza (2018): reportan que los estudiantes: "han presentado un bajo rendimiento académico" (pág. 635); y Pérez y Castro (2018b), señalan que los estudiantes: "se han mostrado desmotivados en los últimos semestres" (pág. 68).

De manera particular, en el ámbito universitario es muy importante el pensamiento computacional porque provee a los estudiantes con el perfil requerido actualmente. Según Socorro (2018), el objetivo de las universidades 
venezolanas es: "formar profesionales integrales con perfiles inter 0 transdisciplinarios, capaces de enfrentar la realidad y aportar respuestas viables y confiables a una sociedad que las requiere" (pág. 287). Por lo tanto, el pensamiento computacional es fundamental por su carácter transdisciplinario en la resolución de problemas, justificando la reflexión sobre otras maneras para el desarrollo del pensamiento computacional en aras de facilitar la apropiación de éste por parte de los estudiantes, lo cual representa el interés de este ensayo.

El estudio de conceptos, características y fases asociadas al pensamiento computacional, sugieren que las habilidades que lo componen pueden ser adquiridas en contextos distintos al aprendizaje de la programación de computadores. En ese orden de ideas, la relación natural entre las habilidades del pensamiento computacional y las actividades de la vida cotidiana, permiten asomar la posibilidad del desarrollo de esas habilidades durante la ejecución de actividades de la vida cotidiana, lo cual representa la propuesta de este ensayo.

Adicionalmente, considerando los resultados satisfactorios obtenidos en estudios que fueron realizados antes de la aparición del concepto del pensamiento computacional, relacionados a la aplicación de la metacognición para fortalecer el proceso de resolución de problemas, donde Doménech (2004a), concluye que: "la solución exitosa se obtiene después de una planificación" (pág. 322); en este ensayo se propone específicamente la utilización de la metacognición para el desarrollo de las habilidades del pensamiento computacional durante la realización de actividades de la vida cotidiana.

La sección de desarrollo de este ensayo se organiza de la siguiente manera: primero, se presentan los aspectos teóricos del pensamiento computacional (conceptos, características y fases); luego, se mencionan ejemplos de aplicación en la vida cotidiana asociados a cada una de las 
habilidades requeridas; y finalmente, se introduce el concepto de metacognición, indicando su posible utilización para el desarrollo de las habilidades del pensamiento computacional durante la ejecución de actividades de la vida cotidiana.

\section{Desarrollo}

El concepto de pensamiento computacional fue planteado por primera vez por Wing (2006a), de la siguiente manera: "representa una actitud y un conjunto de habilidades universalmente aplicables que todos, no solo científicos informáticos, estarían ansiosos por aprender y usar" (pág. 33). Si bien es cierto que este es un concepto muy general que lo concibe como la combinación de una actitud y un conjunto de habilidades, también es cierto que presenta la premisa principal del pensamiento computacional, es decir, que todas las personas pudieran aplicarlo en diferentes ámbitos.

Algunos años más tarde, Aho (2012), especifica un poco más el alcance del pensamiento computacional, refiriéndose a él como un proceso: "Es el proceso de pensamiento involucrado en la formulación de problemas, por lo que sus soluciones se pueden representar como pasos y algoritmos computacionales" (pág. 832). Esta definición clarifica el alcance del pensamiento computacional porque lo enmarca explícitamente en el contexto de la resolución de problemas, sin embargo, deja implícito los ámbitos de aplicación.

Posteriormente, Basogain, Olabe y Olabe (2015), asocian directamente el concepto de pensamiento computacional con las actividades de la vida cotidiana, refiriéndose a éste como: "una metodología basada en la implementación de los conceptos básicos de las ciencias de la computación para resolver problemas cotidianos, diseñar sistemas domésticos y realizar tareas rutinarias" (pág. 3). Esa relación con la vida cotidiana motiva la revisión y reflexión que se hace en este ensayo, es decir, la revisión de las 
características, fases y aplicaciones en la vida cotidiana del pensamiento computacional; y la reflexión sobre la adquisición de sus habilidades inherentes.

Las características del pensamiento computacional según Wing (2006b), son seis: 1). Conceptualización; 2). habilidad básica; 3). manera de pensar; 4). complemento con el pensamiento matemático e ingenieril; 5). Ideas; y 6). para cualquiera en cualquier parte (pág. 35). Las dos primeras características están relacionadas a su naturaleza: la conceptualización hace referencia a que se debe pensar en múltiples niveles de abstracción y no sólo como para programar un computador; y la característica de habilidad básica hace referencia a que no debe ser algo mecánico.

Las dos características siguientes hacen aclaraciones sobre su origen: la característica de manera de pensar se refiere a que las personas son las que piensan y no los computadores, es decir, el pensamiento computacional es una manera en que los seres humanos solucionan problemas con ayuda del computador, por lo tanto, no se trata de que los humanos piensen como los computadores; y por otra parte, la característica de complemento con el pensamiento matemático e ingenieril, hace referencia a que el fundamento matemático e ingenieril son los que hacen posible el pensamiento computacional para la construcción de sistemas informáticos.

Las dos características restantes, relacionadas al alcance del pensamiento computacional, son las que despiertan el interés de este ensayo: la característica de ideas, destaca que el pensamiento computacional no sólo tiene que ver con el software que se produce, sino que está presente a diario en las vidas de las personas; y finalmente, la característica de para cualquiera en cualquier parte, asoma la posibilidad de utilizar este pensamiento permanentemente, incluso como filosofía de vida.

En general, las seis características presentadas, sugieren los siguientes aspectos con respecto a la resolución de problemas en la vida cotidiana: 
primero, el pensamiento computacional no puede ser mecánico debido a las distintas configuraciones de problemas que pudieran presentarse en la vida cotidiana; segundo, las bases de matemáticas e ingeniería son complementos naturales del pensamiento computacional, sin embargo, no restringen totalmente su uso; y tercero, su amplio alcance de aplicación lo hace apto para ser considerado en la resolución de cualquier problema.

Ahora bien, con respecto a las fases del pensamiento computacional, algunos investigadores como Román, Pérez y Jiménez (2015), promueven la existencia de cuatro fases: 1). Descomposición; 2). reconocimiento de patrones; 3). generalización de patrones y abstracción; y 4). diseño algorítmico (pág. 2). La descomposición es la capacidad para fraccionar una tarea en los pasos que la conforman. El reconocimiento de patrones es la capacidad para percibir similitudes, dentro del mismo problema o con otros problemas, que permitan reducir el camino hacia la resolución del problema.

A diferencia, la fase de generalización de patrones y abstracción, requiere de la capacidad para filtrar e ignorar toda la información que no es necesaria para resolver un problema determinado, y de generalizar la que sí es necesaria. Por su parte, la fase de diseño algorítmico requiere la capacidad de desarrollar una estrategia paso a paso, es decir, establecer una secuencia de instrucciones para resolver el problema.

Las cuatro fases presentadas anteriormente representan las principales habilidades que requiere una persona para resolver problemas utilizando el pensamiento computacional. Para el autor de este ensayo, la clave del pensamiento computacional es la primera fase, porque a partir de ésta se hace posible la aplicación de las demás fases, es decir, descomponer un problema conduce al reconocimiento y la generalización de patrones, lo cual inevitablemente converge a la especificación de los pasos que resuelven el problema, es decir, al diseño algorítmico.

En aras de clarificar la relación del pensamiento computacional con la 
vida cotidiana, a continuación, se presentan algunos ejemplos de aplicaciones del pensamiento computacional para cada una de sus habilidades. La habilidad de descomposición se ve reflejada frecuentemente cuando una persona le da indicaciones a otra para llegar a algún lugar, por ejemplo, caminar dos cuadras, girar a la derecha y caminar hasta encontrar la casa verde. Por su parte, la habilidad de reconocimiento de patrones, se puede ver reflejada en los comportamientos de las personas, por ejemplo, cuando los niños naturalmente saben identificar los eventos que enfurecen a sus padres.

Por otra parte, los ejemplos de aplicación de la abstracción y generalización, se reflejan cuando se hacen representaciones de las cosas en función de los atributos de interés para un momento determinado, por ejemplo, al comprar alimentos los criterios de selección entre diferentes marcas de un mismo producto, pueden ser el precio o la calidad, los cuales dependerán de los intereses de los compradores. Para finalizar con los ejemplos de aplicaciones, un ejemplo de diseño algorítmico se puede apreciar claramente cuando un cocinero detalla su propia receta para la preparación de sus comidas.

Ahora bien, como se puede apreciar en los ejemplos, las personas ya utilizan las habilidades asociadas al pensamiento computacional en la vida cotidiana, en mayor o menor medida, dependiendo de la complejidad de las actividades que realizan, es decir, utilizando las habilidades tanto de manera aislada como en combinaciones entre ellas. En ese sentido, la reflexión principal de este ensayo está orientada hacia la posibilidad de desarrollar conscientemente el pensamiento computacional durante la realización de las actividades de la vida cotidiana.

Debido a que Zapata (2015), hace énfasis en la metacognición como una competencia que abarca todas las fases: "podemos considerar un conjunto de competencias necesarias en todas las fases, sin que tengan que ver directamente y de forma exclusiva con algunas de ellas, como puede ser 
la metacognición" (pág. 38). Aunado a que en estudios previos a la aparición del concepto del pensamiento computacional, se ha utilizado la metacognición para fortalecer el proceso de resolución de problemas, donde Doménech (2004b), encontró que: "una elevada metacognición permite regular la resolución exitosa" (pág. 384); en este ensayo se propone la utilización de la metacognición para el desarrollo de las habilidades del pensamiento computacional (descomposición, reconocimiento de patrones, abstracción y generalización, y diseño algorítmico) durante la realización de actividades de la vida cotidiana.

La metacognición según Flavell (1976), hace referencia a dos elementos: primero, el conocimiento que una persona tiene sobre sus propios procesos cognitivos o cualquier otro aspecto relacionado con ellos; y segundo, a la supervisión activa y consecuente regulación de estos procesos (pág. 232). Estos dos elementos son la base de la metacognición, tal como lo indica Zulma (2006), cuando menciona que los investigadores que trabajaron posteriormente con la metacognición han mantenido dos dimensiones: "en la mayoría de las veces estableciendo una mayor precisión en sus componentes, pero que retienen la distinción inicial de dos dimensiones en la metacognición: el conocimiento y la regulación de la cognición" (pág. 123).

Por lo tanto, la propuesta que se hace en este ensayo consiste en asociar las habilidades del pensamiento computacional a esos dos elementos metacognitivos. Por un lado, para el conocimiento que una persona tiene sobre sus propios procesos, debe tomarse conciencia de que las habilidades del pensamiento computacional ya están inmersas en la vida cotidiana y que con frecuencia son utilizadas. Por otra parte, para la supervisión activa, debe considerarse la posibilidad de usar las habilidades del pensamiento computacional durante la ejecución de las actividades de la vida cotidiana.

Para finalizar, en aras de que todos los lectores tengan la posibilidad de desarrollar el pensamiento computacional, se sugiere cultivar el hábito de 
responder cuatro preguntas durante la realización de todas sus actividades cotidianas. Las cuatro preguntas son las siguientes: primera, ¿Estoy descomponiendo la actividad en varias partes?; segunda, ¿Estoy identificando las partes que se repiten o que están presentes en otras actividades?; tercera, ¿Estoy considerando únicamente los elementos de interés?; y cuarta, ¿Estoy diseñando una estrategia para realizar la actividad?.

\section{Conclusiones}

El pensamiento computacional es un concepto de reciente aparición, cuya relevancia como objeto de estudio está soportada por el interés de los sistemas educativos en incorporarlo como eje transversal de todas las disciplinas mediante la enseñanza de la programación de computadores desde tempranas edades. La revisión de las dificultades del aprendizaje de la programación hace necesaria la reflexión sobre nuevas formas de desarrollar el pensamiento computacional.

El estudio de conceptos, características y fases del pensamiento computacional, sugieren la posibilidad de desarrollar este pensamiento durante la realización de actividades de la vida cotidiana, mediante la aplicación de la metacognición. Para ello, sólo dos requisitos son necesarios: primero, tomar conciencia de que ya se vienen utilizando en la vida cotidiana las habilidades del pensamiento computacional (descomposición, reconocimiento de patrones, abstracción y generalización, y algoritmos); y segundo, considerar conscientemente esas habilidades en la realización de las actividades de la vida cotidiana.

En primera instancia, los dos requisitos presentados para desarrollar las habilidades del pensamiento computacional sólo demandan una iniciativa proactiva de las personas para cultivar el hábito de responder las cuatro preguntas durante la realización de todas sus actividades cotidianas: ¿Estoy descomponiendo la actividad en varias partes?; ¿Estoy identificando las partes 
que se repiten o que están presentes en otras actividades?; ¿Estoy considerando únicamente los elementos de interés?; y ¿Estoy diseñando una estrategia para realizar la actividad?.

La iniciativa proactiva mencionada anteriormente representa el primer desafío de esta propuesta para los que promueven la adquisición del pensamiento computacional en las demás personas. Finalmente, es importante destacar que la propuesta metacognitiva presentada en este ensayo, sólo es producto de las reflexiones realizadas, quedando como trabajo futuro la realización de los respectivos experimentos para conocer su efectividad.

\section{Referencias}

Aho, A. (2012). Computation and computational thinking. The Computer Journal, 55(7), 832-835, e-ISSN: 1460-2067. Recuperado de: https://doi.org/10.1093/comjnl/bxs074

Basogain, X., Olabe, M., \& Olabe, J. (2015). Pensamiento Computacional a través de la Programación: Paradigma de Aprendizaje. RED. Revista de Educación a Distancia, (46), 1-33, e-ISSN: 1578-7680. Recuperado de: https://revistas.um.es/red/article/view/240011

Compañ P., Satorre R., Llorens, F., \& Molina, R. (2015). Enseñando a programar: un camino directo para desarrollar el pensamiento computacional. RED. Revista de Educación a Distancia, (46), 1-15, ee-ISSN: 1578-7680. Recuperado de:

https://revistas.um.es/red/article/view/240191

Doménech, M. (2004a,b). El papel de la inteligencia y de la metacognición en la resolución de problemas. Tesis Doctoral. España: Universitat Rovira i Virgili. Recuperado de:

https://dialnet.unirioja.es/servlet/tesis?codigo=8216

Flavell, J. (1976). Metacognitive aspects of problem solving. The nature of 
intelligence, 231-235, ISBN: 04700-13842. Hillsdale, New Jersey: Lawrence Erlbaum Associates.

Ortega, B., \& Brouard, M. (2018). Robótica DIY: pensamiento computacional para mejorar la resolución de problemas. RELATEC. Revista Latinoamericana de Tecnología Educativa, 17(2), 129-143, e-ISSN: 1695-288X. Recuperado de:

https://doi.org/10.17398/1695-288X.17.2.129

Pérez, J., \& Castro, J. (2018a). Estímulo del razonamiento lógico mediante el juego Millonario en C para la asignatura "Programación 1". tekhné, 21(3), 8-15, e-ISSN: 1316-39630. Recuperado de:

http://revistasenlinea.saber.ucab.edu.ve/temas/index.php/tekhne/articl e/view/3804

Pérez, J., \& Castro, J. (2018b). LRS1: Un robot social de bajo costo para la asignatura "Programación 1". RCTA. Revista Colombiana de Tecnologías de Avanzada, 2(32), 68-77, e-ISSN: 1692-7257. Recuperado de:

http://revistas.unipamplona.edu.co/ojs viceinves/index.php/RCTA/articl e/view/3028

Pérez, J., \& Pedroza, O. (2018). LM1: una metodología de estudio para la asignatura "Programación 1". Educere, 22(73), 635-648, e-ISSN: 1316-4910. Recuperado de:

https://dialnet.unirioja.es/servlet/articulo?codigo $=6651822$

Román, M., Pérez, J., \& Jiménez, C. (2015). Test de pensamiento computacional: diseño y psicometría general. Madrid, España: III Congreso Internacional sobre Aprendizaje, Innovación y Competitividad (CINAIC). Recuperado de:

https://doi.org/10.13140/Rg.2.1.3056.5521

Socorro, M. (2018). Transdisciplinariedad: Una Mirada desde la Educación Universitaria. Revista Scientific, 3(10), 278-289, e-ISSN: 2542-2987. 
Recuperado de:

https://doi.org/10.29394/Scientific.issn.2542-2987.2018.3.10.15.278-

$\underline{289}$

Trilling, B., \& Fadel, C. (2009). 21st Century Skills: Learning for Life in Our Times. Primera edición, ISBN: 978-0-470-47538-6. San Francisco, C.A.: John Wiley \& Sons.

Wing, J. (2006a,b). Computational Thinking. Communications of the ACM, 49(3), 33-35, e-ISSN: 1557-7317. Recuperado de:

https://doi.org/10.1145/1118178.1118215

Zapata, M. (2015). Pensamiento computacional: Una nueva alfabetización digital. RED. Revista de educación a distancia, (46), 1-47, e-ISSN: 1578-7680. Recuperado de:

https://revistas.um.es/red/article/view/240321

Zulma, M. (2006). Aprendizaje autorregulado: el lugar de la cognición, la metacognición y la motivación. Estudios Pedagógicos, 32(2), 121132, e-ISSN: 0716-050X. Recuperado de:

https://www.redalyc.org/articulo.oa?id=173514131006 


\section{Jesús Alberto Pérez Angulo}

e-mail: jesuspangulo@ula.ve

Nací en Mérida, estado Mérida, Venezuela, el 28 de

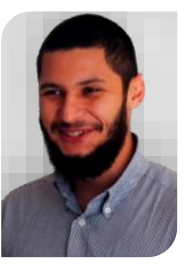
octubre del año 1989. Ingeniero Electrónico (2012); Ingeniero de Sistemas (2014); Magister en Educación Superior Mención Docencia Universitaria (2015); estudiante de Doctorado en Ciencias de la Educación (2015 - presente). Actualmente soy Profesor de la Escuela de Ingeniería de Sistemas de la Universidad de Los Andes (ULA) e Integrante del Laboratorio de Sistemas Discretos, Automatización e Integración. Mis líneas de investigación son: Interacción Humano-Robot y Enseñanza de la Ingeniería.

El contenido de este manuscrito se difunde bajo una Licencia de Creative Commons ReconocimientoNoComercial-Compartirlgual 4.0 Internacional 